\title{
Effect of Corrugation and Reinforcement on the Dispersion of SH-wave Propagation in Corrugated Poroelastic Layer Lying over a Fibre-reinforced Half-space
}

\author{
Abhishek Kumar SINGH, Amrita DAS, Anirban LAKSHMAN, \\ and Amares CHATTOPADHYAY
}

Indian Institute of Technology (Indian School of Mines), Dhanbad, Jharkhand, India e-mails: abhi.5700@gmail.com, amritadas.ism@gmail.com (corresponding author), anirban.ism@gmail.com, amares.c@gmail.com

\section{Abstract}

The presence of porosity and reinforcement in a medium is an important factor affecting seismic wave propagation and plays vital role in many geophysical prospects. Also, the presence of salt and ore deposits, mountains, basins, mountain roots, etc. is responsible for the existence of corrugated boundary surfaces of constituent layers. Such facts brought motivation for the present paper which deals with the propagation of $\mathrm{SH}$ wave in a heterogeneous fluid-saturated poroelastic layer with corrugated boundaries lying over an initially stressed fibre-reinforced elastic halfspace. Closed form of dispersion relation has been obtained and is found in well agreement to classical Love wave equation for isotropic case. The effect of corrugation, wave number, undulation, position parameter, horizontal compressive/tensile initial stress and heterogeneity on phase velocity has been analysed through numerical computation and graphical illustration. Moreover, comparative study exploring the effect of presence and absence of reinforcement in half-space on dispersion curve is the major highlight of the current study.

Key words: corrugated boundary, initial stress, heterogeneous poroelastic, reinforcement, $S H$-wave. 


\section{INTRODUCTION}

A material containing pores which are generally filled with fluid is said to be a porous material. The skeletal portion of the material is usually known as a frame. A shear wave propagating in the frame with an inertial contribution from the pore fluid was predicted in Biot's theory. Moreover, Biot (1962) also discussed the theories of consolidation and elastic-wave propagation in fluid-saturated porous media. Due to bedding, compaction and presence of aligned micro cracks, the saturated porous materials are anisotropic. Sandstone, water-saturated ocean sediments and other sediments permeated by oil, present below the Earth's surface, are liquid-saturated porous materials. The study regarding porous media find its application in the field of applied science and engineering including filtration, acoustics, soil rock mechanics, construction engineering, hydrogeology, biophysics and material science. Apart from these, the dynamic behaviour of structured porous media is of great concern in the arena of seismology, earthquake engineering and fluid dynamics. A number of papers have been published taking into view the importance of study of porous media. Ke et al. $(2005,2006)$ studied the propagation of the Love wave in an inhomogeneous fluid-saturated porous layered half-space with the elastic constants varying as functions of depth. Son and Kang (2012) elucidated the propagation of shear wave in a poroelastic layer constrained between two elastic layers. Chattaraj et al. (2013) investigated the propagation of Love wave in an initially stressed fluid-saturated anisotropic porous layer with an irregular boundary sandwiched between two isotropic half-spaces. Samal and Chattaraj (2011) obtained the dispersion relation in the form of a ninth order determinant for the propagation of surface wave in fibre-reinforced anisotropic elastic layer between a liquidsaturated porous half-space and uniform liquid layer. Some important results were presented by Chattopadhyay and Choudhury $(1990,1995)$ and Sengupta and Nath (2001) based on the study on the propagation of seismic waves in reinforced medium.

In light of the problems of elastic stability for anisotropic media, the study of fibre-reinforced layer is found to be very crucial. A medium may possess an intrinsic anisotropy or it may be isotropic for finite deformations and may exhibit an induced anisotropy for incremental deformations in the vicinity of a state of initial stress. Due to the appearance of new features concerned with the phenomena of internal instability, more analysis is needed to study the problems of anisotropic media compared to the problems of isotropic media.

A layer of fibre-reinforced media may also be present inside the Earth which are formed because of high initial stress. The characteristics of the fibre-reinforced material are entirely different from that of its constituent ma- 
terials, namely the concrete and steel. The individual components remain isolated within the composite materials, i.e., the components are bound together so that there could be no relative displacements between them as long as they remain in elastic condition. The fibre-reinforced composite comprises of the fibre, the matrix and the interface between the ingredient materials. The fibres form the dispersed phase while the matrix forms the continuous phase. Under certain temperature, pressure and high initial stress, some fibre materials get modified to self-reinforced fibres. The Earth crust may contain some hard/soft rocks or material that may exhibit self-reinforced properties. Carbon, nylon or conceivable metal whiskers, etc., are examples of fibrereinforced materials. The propagation of seismic wave through reinforced media is of great importance due to its application in mining and civil engineering, geophysical prospecting, soil mechanics and architecture. Singh and Singh (2004) examined the propagation of plane waves in fibre-reinforced elastic media and showed that the phase velocities of quasi $P$ - and $S V$-waves depend on the angle between direction of propagation and the direction of reinforcement. They also discussed the reflection of these elastic waves from the free surface of a fibre-reinforced elastic half-space. Recently, Chattopadhyay et al. (2013, 2010a, b) and Chattopadhyay and Singh (2012a, b) attempted to investigate the effect of reinforcement on the propagation of magnetoelastic shear waves, torsional surface waves and crack.

Earth is a layered structure, constituted of layers of different kinds of materials with divergent properties. Some physical factors involving overburdened layer, atmospheric pressure, variation in temperature, slow process of creep and gravitational field give rise to a large amount of initial stress in a medium. The rigidity of the medium alters due to the presence of initial stress in it. More precisely, the presence of tension in a medium supports more rigidity, whereas the presence of compression in a medium supports less rigidity. Therefore, initial stress has noteworthy impact on the propagation of seismic waves, which made its study obligatory. An analytical approach was adopted by $\mathrm{Du}$ et al. (2007) to investigate Love wave propagation in layered magneto-electro-elastic structures with initial stress, where a thin layer of piezomagnetic (piezoelectric) material is bonded to a semi-infinite piezoelectric (piezomagnetic) substrate. Chattopadhyay et al. (2010c) scrutinized the propagation of shear waves in viscoelastic medium at irregular boundaries. Many attempts have been made to study the effect of corrugated boundaries on reflection and refraction of seismic waves, but the effect of corrugated boundaries on the propagation of seismic waves through mediums pertaining to various incredible features still needs to be investigated.

In most of the real situations, the surface of the layers as well as the boundaries separating the different layers in the interior of the Earth are not 
always regular. Therefore, the study of propagation of waves in elastic media with corrugated boundary plays an important role for better understanding and the prediction of seismic wave behaviour at continental margins and mountain roots. The corrugation in the media may also arise due to salt and ore deposits beneath the Earth's surface. There are possibilities that the boundary surface of the layers is of undulatory nature. Kundu et al. (2014) discussed the dispersion of Love wave in pre-stressed homogeneous medium over a porous half-space with irregular boundary surfaces. The problem of the propagation of a Love wave in a corrugated isotropic layer over a homogeneous isotropic half-space has been investigated by Singh (2011). The study of the scattered field which results when a Love wave is incident on a layer having an irregular surface has been furnished by Wolf (1970). Tomar and Kaur (2007) studied the $S H$-waves at a corrugated interface between a dry sandy half-space and an anisotropic elastic half-space. Singh and Tomar (2008) demonstrated the $q P$-wave at a corrugated interface between two dissimilar pre-stressed elastic half-spaces using Rayleigh's method of approximation. Kaur et al. (2005) illustrated the reflection and refraction of SHwaves at a corrugated interface between two laterally and vertically heterogeneous viscoelastic solid half-spaces.

The present paper deals with the effect of corrugated boundary surfaces on the dispersion of $\mathrm{SH}$-wave in a corrugated heterogeneous fluid-saturated poroelastic layer overlying an initially stressed fibre-reinforced elastic halfspace. The elastic constants and density of heterogeneous fluid-saturated poroelastic layer are assumed to vary exponentially in terms of space variable pointing vertically downward. The dispersion relation has been obtained in closed form and found in well-agreement to the classical Love wave equation as a particular case of the problem. The effect of presence and absence of heterogeneity, porosity, and corrugated boundary surfaces of the layer, horizontal compressive/tensile initial stress and reinforcement existing in the elastic half-space, and anisotropy of both layer and semi-infinite medium are presented with the aid of particular cases computed numerically and illustrated graphically.

\section{GEOMETRY OF THE PROBLEM}

We consider a heterogeneous porous layer of average finite thickness $h$ lying over a fibre-reinforced half-space under initial stress. Rectangular cartesian co-ordinate system has been taken into consideration with origin $O$ at the corrugated interface between the layer and half-space. The heterogeneity in the upper porous layer is caused due to exponential variation of space variable which is pointing positively downwards. The $x_{1}$-axis, being the direction of wave propagation, and the $x_{2}$-axis are taken in the horizontal plane 


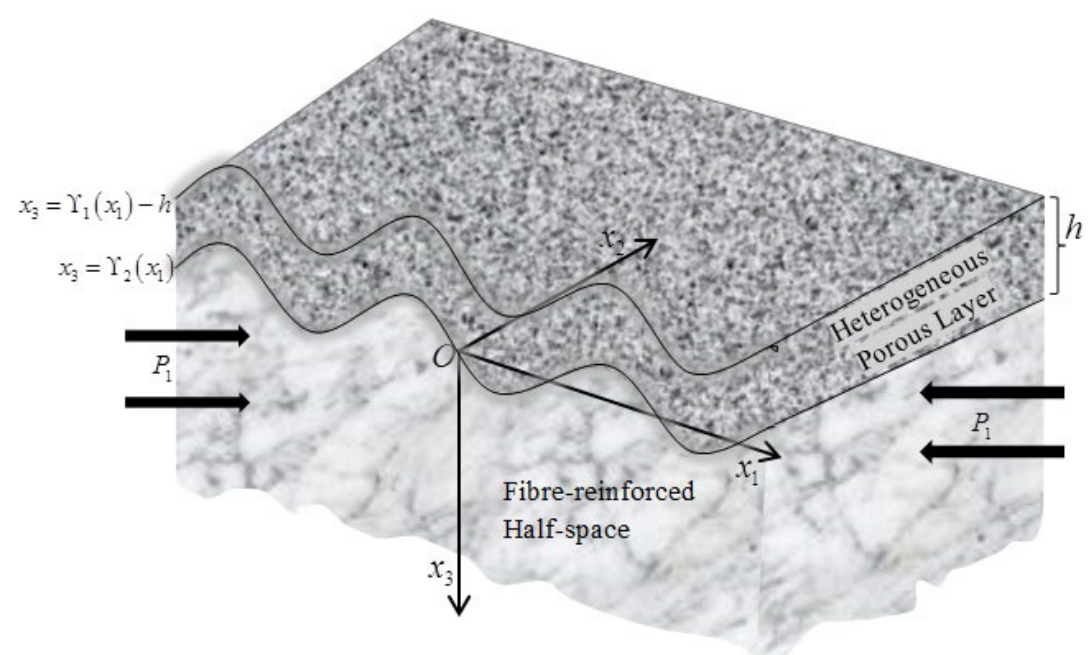

Fig. 1. Geometrical model of the problem.

of rectangular co-ordinate system, and the $x_{3}$-axis is vertically downwards. The said geometry is shown in Fig. 1.

Let the equation of uppermost corrugated boundary surface be $x_{3}=$ $\Upsilon_{1}\left(x_{1}\right)-h$ and the equation of corrugated interface between layer and halfspace be $x_{3}=\Upsilon_{2}\left(x_{1}\right)$, where $\Upsilon_{1}\left(x_{1}\right)$ and $\Upsilon_{2}\left(x_{1}\right)$ are periodic functions and independent of $x_{2}$. Taking a suitable origin of coordinates we can represent the trigonometric Fourier series of $\Upsilon_{1}\left(x_{1}\right), \Upsilon_{2}\left(x_{1}\right)$ as follows (Asano 1966):

$$
\Upsilon_{n}=\sum_{l=1}^{\infty}\left[\Upsilon_{l}^{(n)} e^{i l \Phi x_{1}}+\Upsilon_{-l}^{(n)} e^{-i l \Phi x_{1}}\right], \quad n=1,2,
$$

where $\Upsilon_{l}^{(n)}$ and $\Upsilon_{-l}^{(n)}$ are Fourier expansion coefficients and $l$ is series expansion order. Let us introduce the constants $a, b, R_{l}^{(n)}, I_{l}^{(n)}$ as follows:

$$
\Upsilon_{ \pm 1}^{(1)}=\frac{a}{2}, \Upsilon_{ \pm 1}^{(2)}=\frac{b}{2}, \Upsilon_{ \pm l}^{(n)}=\frac{R_{l}^{(n)} \mp i I_{l}^{(n)}}{2}, n=1,2, \text { and } l=2,3, \ldots
$$

and

$$
\begin{aligned}
& \Upsilon_{1}=a \cos \Phi x_{1}+R_{2}^{(1)} \cos 2 \Phi x_{1}+I_{2}^{(1)} \sin 2 \Phi x_{1}+\ldots+R_{l}^{(1)} \cos l \Phi x_{1}+I_{l}^{(1)} \sin l \Phi x_{1}+\ldots \\
& \Upsilon_{2}=b \cos \Phi x_{1}+R_{2}^{(2)} \cos 2 \Phi x_{1}+I_{2}^{(2)} \sin 2 \Phi x_{1}+\ldots+R_{l}^{(2)} \cos l \Phi x_{1}+I_{l}^{(2)} \sin l \Phi x_{1}+\ldots
\end{aligned}
$$

where $R_{l}^{(n)}, I_{l}^{(n)}$ are the cosine and sine Fourier coefficients, respectively. As far the present problem is concerned, the corrugated upper boundary surface 
and lower boundary surface may be expressed with the aid of cosine terms, i.e., $\Upsilon_{1}=a \cos \Phi x_{1}$ and $\Upsilon_{2}=b \cos \Phi x_{1}$, respectively, where $\Phi$ is the wave number, $a$ and $b$ are the amplitudes of corrugation and the wavelength of the corrugation is $2 \pi / \Phi$.

Let us assume $\left(u_{1}, u_{2}, u_{3}\right)$ and $\left(U_{1}, U_{2}, U_{3}\right)$ as the displacement components of the solid part and liquid part of the porous aggregate, respectively, in the upper corrugated heterogeneous porous layer. Further, we assume $\left(v_{1}, v_{2}, v_{3}\right)$ to be the displacement component of lower fibre-reinforced halfspace under initial stress.

\section{GOVERNING EQUATIONS AND SOLUTION OF THE PROBLEM}

For the propagation of $\mathrm{SH}$-wave, we consider

$$
\begin{aligned}
& u_{1}=0, u_{3}=0, u_{2}=u_{2}\left(x_{1}, x_{3}, t\right), \\
& U_{1}=0, U_{3}=0, U_{2}=U_{2}\left(x_{1}, x_{3}, t\right), \\
& v_{1}=0, v_{3}=0, v_{2}=v_{2}\left(x_{1}, x_{3}, t\right) .
\end{aligned}
$$

\subsection{Dynamics of the upper corrugated heterogeneous fluid-saturated poroelastic layer}

The stress-strain relation for the transversely isotropic fluid-saturated poroelastic layer may be expressed as (Biot 1965)

$$
\left[\begin{array}{c}
\sigma_{11} \\
\sigma_{22} \\
\sigma_{33} \\
\sigma_{12} \\
\sigma_{23} \\
\sigma_{31} \\
\sigma
\end{array}\right]=\left[\begin{array}{ccccccc}
2 B_{1}+B_{2} & B_{2} & B_{3} & 0 & 0 & 0 & B_{6} \\
B_{2} & 2 B_{1}+B_{2} & B_{3} & 0 & 0 & 0 & B_{6} \\
B_{3} & B_{3} & B_{4} & 0 & 0 & 0 & B_{7} \\
0 & 0 & 0 & 2 B_{1} & 0 & 0 & 0 \\
0 & 0 & 0 & 0 & 2 B_{5} & 0 & 0 \\
0 & 0 & 0 & 0 & 0 & 2 B_{5} & 0 \\
B_{6} & B_{6} & B_{7} & 0 & 0 & 0 & B_{8}
\end{array}\right]\left[\begin{array}{c}
e_{11} \\
e_{22} \\
e_{33} \\
e_{12} \\
e_{23} \\
e_{31} \\
\varsigma
\end{array}\right],
$$

where $\sigma_{i j}$ are the total stress components in the solid skeleton of the porous material and $e_{i j}$ are the components of strain acting on the solid phase of the porous material. $B_{1}$ to $B_{8}$ are the material constants and $\zeta$ is the fluid volumetric strain. $\sigma$ is the fluid pore pressure acting on the fluid phase of the porous material and it can be written as $\sigma=-\phi p$, where $\phi$ is the porosity and $p$ is the pressure in the fluid existing in the porous layer. $e_{i j}$ and $\zeta$ may be written as

$$
e_{i j}=\frac{1}{2}\left(u_{i, j}+u_{j, i}\right), \quad \varsigma=\nabla \cdot U_{i}
$$


The basic dynamic relation for wave propagation in fluid-saturated porous layer without body force and viscosity, given by Biot (1965), is

$$
\begin{gathered}
\sigma_{i j, j}=\frac{\partial^{2}}{\partial t^{2}}\left(\rho_{11} u_{i}+\rho_{12} U_{i}\right), \\
\sigma_{, i}=\frac{\partial^{2}}{\partial t^{2}}\left(\rho_{12} u_{i}+\rho_{22} U_{i}\right),
\end{gathered}
$$

where $\rho_{11}, \rho_{12}$, and $\rho_{22}$ are mass coefficients. These mass coefficients are taken into account for the inertias of the solid and fluid phases which are related to the mass density of solid $\left(\rho_{s}\right)$ and fluid $\left(\rho_{f}\right)$ as

$$
\begin{aligned}
& \rho_{11}+\rho_{12}=(1-\phi) \rho_{s}, \\
& \rho_{12}+\rho_{22}=\phi \rho_{f}, \\
& \rho_{1}=\rho_{11}+2 \rho_{12}+\rho_{22}=\rho_{s}+\phi\left(\rho_{f}+\rho_{s}\right) .
\end{aligned}
$$

where $\rho_{1}$ is the total mass density of solid-liquid aggregate.

Moreover, the mass coefficients satisfy the following inequalities:

$$
\rho_{11}>0, \rho_{12}<0, \rho_{22}>0, \rho_{11} \rho_{22}-\rho_{12}^{2}>0 . .
$$

Combining Eqs. 2 and 5, we have

$$
\begin{aligned}
& \frac{\partial \sigma_{23}}{\partial x_{3}}+\frac{\partial \sigma_{12}}{\partial x_{1}}=\frac{\partial^{2}}{\partial t^{2}}\left(\rho_{11} u_{2}+\rho_{12} U_{2}\right), \\
& 0=\frac{\partial^{2}}{\partial t^{2}}\left(\rho_{12} u_{2}+\rho_{22} U_{2}\right) .
\end{aligned}
$$

Now, the heterogeneity in the fluid-saturated porous layer is considered as

$$
B_{1}=\tilde{B}_{1} e^{\alpha_{1}\left(x_{3}+h\right)}, B_{5}=\tilde{B}_{5} e^{\alpha_{1}\left(x_{3}+h\right)}, \rho_{1}=\tilde{\rho}_{1} e^{\alpha_{1}\left(x_{3}+h\right)},
$$

where $\alpha_{1}$ is the heterogeneity parameter having dimension inverse of length, acting in the upper corrugated layer. $\tilde{B}_{1}, \tilde{B}_{5}$, and $\tilde{\rho}_{1}$ are the values of $B_{1}, B_{5}$, and $\rho_{1}$, respectively, at the uppermost surface when $x_{3}=-h$.

Using Eqs. 3 and 8, Eq. 7 lead to

$$
\tilde{B}_{1} e^{\alpha_{1}\left(x_{3}+h\right)} \frac{\partial^{2} u_{2}}{\partial x_{1}^{2}}+\tilde{B}_{5} e^{\alpha_{1}\left(x_{3}+h\right)} \frac{\partial^{2} u_{2}}{\partial x_{3}^{2}}+\alpha_{1} \tilde{B}_{5} e^{\alpha_{1}\left(x_{3}+h\right)} \frac{\partial u_{2}}{\partial x_{3}}=d_{1} \frac{\partial^{2} u_{2}}{\partial t^{2}},
$$

where $d_{1}=\rho_{11}-\frac{\rho_{12}^{2}}{\rho_{22}}$. 
Introducing $\gamma_{11}=\frac{\rho_{11}}{\tilde{\rho}_{1}}, \gamma_{12}=\frac{\rho_{12}}{\tilde{\rho}_{1}}$, and $\gamma_{22}=\frac{\rho_{22}}{\tilde{\rho}_{1}}$ Eq. 9 can be rewritten as

$$
\frac{\tilde{B}_{1}}{\tilde{B}_{5}} \frac{\partial^{2} u_{2}}{\partial x_{1}^{2}}+\frac{\partial^{2} u_{2}}{\partial x_{3}^{2}}+\alpha_{1} \frac{\partial u_{2}}{\partial x_{3}}=\frac{d_{1}^{\prime}}{c_{1}^{2}} \frac{\partial^{2} u_{2}}{\partial t^{2}}
$$

where $c_{1}=\sqrt{\frac{\tilde{B}_{1}}{\tilde{\rho}_{1}}}$ and $d_{1}^{\prime}=\gamma_{11}-\frac{\gamma_{12}^{2}}{\gamma_{22}}$.

The solution of Eq. 10 may be taken as

$$
u_{2}\left(x_{1}, x_{3}, t\right)=u_{2}^{*}\left(x_{3}\right) e^{i k\left(x_{1}-c t\right)}
$$

where $k$ is the wave number and $c$ is the common wave velocity.

With the help of Eq. 11, Eq. 10 takes the form

$$
\frac{d^{2} u_{2}^{*}}{d x_{3}^{2}}+\alpha_{1} \frac{d u_{2}^{*}}{d x_{3}}+k^{2}\left(\frac{d_{1}^{\prime} c^{2}}{c_{1}^{2}}-\frac{\tilde{B}_{1}}{\tilde{B}_{5}}\right) u_{2}^{*}=0 .
$$

Therefore, the solution of Eq. 12 may be written as

$$
u_{2}^{*}=e^{-\frac{\alpha_{1} x_{3}}{2}}\left(A \cos p_{1} x_{3}+B \sin p_{1} x_{3}\right),
$$

where $A, B$ are arbitrary constants and $p_{1}=\frac{1}{2} \sqrt{4 k^{2}\left(\frac{d_{1}^{\prime} c^{2}}{c_{1}^{2}}-\frac{\tilde{B}_{1}}{\tilde{B}_{5}}\right)-\alpha_{1}^{2}}$.

Hence, from Eqs. 11 and 13, we have

$$
u_{2}\left(x_{1}, x_{3}, t\right)=e^{-\frac{\alpha_{1} x_{3}}{2}}\left(A \cos p_{1} x_{3}+B \sin p_{1} x_{3}\right) e^{i k\left(x_{1}-c t\right)} .
$$

\subsection{Dynamics of the lower initially stressed fibre-reinforced elastic half- space}

The constitutive equation for a fibre-reinforced linearly elastic anisotropic medium is given by (Belfield et al. 1983)

$$
\begin{aligned}
\tau_{i j} & =\lambda e_{k k} \delta_{i j}+2 \mu_{T} e_{k k}+\alpha\left(a_{k} a_{m} e_{k m} \delta_{i j}+a_{i} a_{j} e_{k k}\right)+2\left(\mu_{L}-\mu_{T}\right)\left(a_{i} a_{k} e_{k j}+a_{j} a_{k} e_{k i}\right) \\
& +\beta a_{k} a_{m} e_{k m} a_{i} a_{j}, \quad i, j, k, m=1,2,3,
\end{aligned}
$$

where $\tau_{i j}$ are stress components, $e_{i j}=\frac{1}{2}\left(\frac{\partial v_{i}}{\partial x_{j}}+\frac{\partial v_{j}}{\partial x_{i}}\right)$ are components of infinitesimal strain, $\delta_{i j}$ is the Kronecker delta, $\vec{a}=\left(a_{1}, a_{2}, a_{3}\right)$ is the preferred 
direction of reinforcement such that $a_{1}^{2}+a_{2}^{2}+a_{3}^{2}=1$. The vector $\vec{a}$ may be a function of position. Indices take the values 1, 2, 3 and summation convention is employed. $\alpha, \beta$ and $\left(\mu_{L}-\mu_{T}\right)$ are reinforcement parameters. $\mu_{T}$ can be identified as the shear modulus in transverse shear across the preferred direction, and $\mu_{L}$ as the shear modulus in longitudinal shear in the preferred direction. $\alpha, \beta$ are specific stress components to take into account different layers for concrete part of the composite material, and $\lambda$ is Lame's constant of elasticity.

The only non-vanishing equation of motion for the propagation of $\mathrm{SH}$ wave keeping in view Eq. 2 and the influence of initial stress $\left(P_{1}\right)$ is obtained as (Biot 1965)

$$
\frac{\partial \tau_{21}}{\partial x_{1}}+\frac{\partial \tau_{23}}{\partial x_{3}}-\frac{P_{1}}{2} \frac{\partial \omega_{21}}{\partial x_{1}}=\rho_{2} \frac{\partial^{2} v_{2}}{\partial t^{2}}
$$

where

$$
\begin{aligned}
& \tau_{12}=\left[\mu_{T} \frac{\partial v_{2}}{\partial x_{1}}+\left(\mu_{L}-\mu_{T}\right) a_{1}\left(a_{1} \frac{\partial v_{2}}{\partial x_{1}}+a_{3} \frac{\partial v_{2}}{\partial x_{3}}\right)\right], \\
& \tau_{23}=\left[\mu_{T} \frac{\partial v_{2}}{\partial x_{3}}+\left(\mu_{L}-\mu_{T}\right) a_{3}\left(a_{1} \frac{\partial v_{2}}{\partial x_{1}}+a_{3} \frac{\partial v_{2}}{\partial x_{3}}\right)\right],
\end{aligned}
$$

and $\omega_{i j}=\frac{1}{2}\left(\frac{\partial v_{i}}{\partial x_{j}}-\frac{\partial v_{j}}{\partial x_{i}}\right)$ are rotational components in the half-space.

In view of Eqs. 15 and 16 leads to

$$
P \frac{\partial^{2} v_{2}}{\partial x_{3}^{2}}+\left(Q+\xi_{1}\right) \frac{\partial^{2} v_{2}}{\partial x_{1}^{2}}+R \frac{\partial^{2} v_{2}}{\partial x_{1} \partial x_{3}}=\frac{1}{c_{2}^{2}} \frac{\partial^{2} v_{2}}{\partial t^{2}},
$$

where

$$
P=1+\left(\frac{\mu_{L}}{\mu_{T}}-1\right) a_{3}^{2}, Q=1+\left(\frac{\mu_{L}}{\mu_{T}}-1\right) a_{1}^{2}, R=2 a_{1} a_{3}\left(\frac{\mu_{L}}{\mu_{T}}-1\right), \xi_{1}=\frac{P_{1}}{2 \mu_{T}}, c_{2}=\sqrt{\frac{\mu_{T}}{\rho_{2}}} .
$$

Let us assume the harmonic wave solution of Eq. 17 in the form

$$
v_{2}\left(x_{1}, x_{3}, t\right)=V_{2}\left(x_{3}\right) e^{i k\left(x_{1}-c t\right)},
$$

$k$ being the wave number, and $c$ is the common wave velocity. Eq. 17 when substituted upon by Eq. 18, takes the form 


$$
\frac{d^{2} V_{2}}{d x_{3}^{2}}+\left(\frac{i k R}{P}\right) \frac{d V_{2}}{d x_{3}}-\frac{1}{P}\left\{\left(Q+\xi_{1}\right) k^{2}-\frac{c^{2} k^{2}}{c_{2}^{2}}\right\} V_{2}=0 .
$$

The solution of Eq. 19 gives the expression for the non-vanishing displacement component of half-space as

$$
v_{2}\left(x_{1}, x_{3}, t\right)=C e^{-p_{2} x_{3}} e^{i k\left(x_{1}-c t\right)},
$$

where $\quad p_{2}=\frac{i k R}{2 P}+k \sqrt{\left(\frac{Q+\xi_{1}}{P}\right)-\frac{R^{2}}{4 P^{2}}-\frac{c^{2}}{c_{2}^{2} P}}=p_{r e}+i p_{i m} \quad$ and $C$ is an arbitrary constant.

\section{BOUNDARY CONDITIONS AND DISPERSION RELATION}

The boundary conditions at the upper corrugated surface of the layer and the common corrugated interface between the layer and the half-space are as follows:

(i) Upper corrugated surface is traction free, i.e.,

$$
\sigma_{23}-\Upsilon_{1}^{\prime} \sigma_{12}=0 \text { at } x_{3}=\Upsilon_{1}\left(x_{1}\right)-h .
$$

(ii) Displacements are continuous at the common corrugated interface of the layer and half-space, i.e.,

$$
u_{2}=v_{2} \text { at } x_{3}=\Upsilon_{2}\left(x_{1}\right) .
$$

(iii) Stresses are continuous at the common corrugated interface of the layer and half-space, i.e.,

$$
\sigma_{23}-\Upsilon_{2}^{\prime} \sigma_{12}=\tau_{23}-\Upsilon_{2}^{\prime} \tau_{12} \text { at } x_{3}=\Upsilon_{2}\left(x_{1}\right),
$$

where $\Upsilon_{1}^{\prime}$ and $\Upsilon_{2}^{\prime}$ are the derivatives of $\Upsilon_{1}$ and $\Upsilon_{2}$, respectively, with respect to $x_{1}$.

Now, with the help of Eqs. 14 and 20, boundary conditions (i), (ii), (iii), respectively, give

$$
\begin{gathered}
A\left[T_{1} \cos \left(p_{1}\left(\Upsilon_{1}-h\right)\right)+T_{2} \sin \left(p_{1}\left(\Upsilon_{1}-h\right)\right)\right] \\
+B\left[T_{1} \sin \left(p_{1}\left(\Upsilon_{1}-h\right)\right)-T_{2} \cos \left(p_{1}\left(\Upsilon_{1}-h\right)\right)\right]=0, \\
A\left[T_{3} \cos \left(p_{1} \Upsilon_{2}\right)+T_{2} \sin \left(p_{1} \Upsilon_{2}\right)\right]+B\left[T_{3} \sin \left(p_{1} \Upsilon_{2}\right)-T_{2} \cos \left(p_{1} \Upsilon_{2}\right)\right]=C e^{-\left(\frac{\alpha_{1}}{2}+p_{2}\right) \Upsilon_{2}-\alpha_{1} h} T_{4},
\end{gathered}
$$




$$
A \cos \left(p_{1} \Upsilon_{2}\right)+B \sin \left(p_{1} \Upsilon_{2}\right)=C e^{\left(\frac{\alpha_{1}}{2}-p_{2}\right) \Upsilon_{2}} .
$$

where the introduced symbols $T_{1}, T_{2}, T_{3}, T_{4}$ are provided in Appendix I.

Eliminating arbitrary constants from Eqs. 21, 22, and 23, we get

$$
\tan \left(p_{1}\left(\Upsilon_{2}-\Upsilon_{1}+h\right)\right)=\frac{T_{2} T_{3}+T_{1} T_{2}-T_{2} T_{4} e^{-\alpha_{1}\left(\Upsilon_{2}+h\right)}}{T_{1} T_{4} e^{-\alpha_{1}\left(\Upsilon_{2}+h\right)}-T_{2}^{2}-T_{1} T_{3}} .
$$

The right-hand side of Eq. 24 is a complex expression. Therefore, comparison of real part on both sides of Eq. 24 gives

$$
\tan \left(p_{1}\left(\Upsilon_{2}-\Upsilon_{1}+h\right)\right)=\frac{\Omega_{1} \Omega_{3}+\Omega_{2} \Omega_{4}}{\Omega_{3}^{2}+\Omega_{4}^{2}},
$$

where $\Omega_{1}, \Omega_{2}, \Omega_{3}, \Omega_{4}$ are provided in Appendix I.

Equation 25 represents the dispersion relation for the propagation of $\mathrm{SH}$ wave in a corrugated heterogeneous porous layer lying over a fibrereinforced half-space under initial stress.

The factor $\left(1-d_{1}^{\prime}\right)$ represents the fraction of porosity in the layer. In light of this, we summarize the following:

(i) For the case when the layer becomes non-porous, we have $\phi \rightarrow 0$, which gives $\rho_{s} \rightarrow \tilde{\rho}_{1}$. Therefore, $\gamma_{11}+\gamma_{12} \rightarrow 1$ and $\gamma_{12}+\gamma_{22} \rightarrow 0$ which leads to $\gamma_{11}-\frac{\gamma_{12}^{2}}{\gamma_{22}} \rightarrow 1$, i.e., $d_{1}^{\prime} \rightarrow 1$.

(ii) In case when the layer becomes a fluid, i.e., $\phi \rightarrow 1$ and hence $\rho_{f} \rightarrow \tilde{\rho}_{1}$, the $S H$-wave velocity in layer cannot exist, which so happens when $d_{1}^{\prime} \rightarrow 0$.

(iii) The layer is said to be porous for the case when $0<d_{1}^{\prime}<1$.

\section{SPECIAL CASE AND VALIDATION}

\section{Case 5.1}

When the layer is bounded by an upper planar surface, i.e., $x_{3}=-h$ for $\square_{1}=0$ and a lower corrugated surface, i.e., $\square_{2}=b \cos \Phi x_{1}$, the dispersion relation 25 reduces to

$$
\tan \left(p_{1}\left(b \cos \Phi x_{1}+h\right)\right)=\frac{\Omega_{1}^{(0)} \Omega_{3}^{(0)}+\Omega_{2}^{(0)} \Omega_{4}^{(0)}}{\left(\Omega_{3}^{(0)}\right)^{2}+\left(\Omega_{4}^{(0)}\right)^{2}},
$$


where $\Omega_{1}^{(0)}, \Omega_{3}^{(0)}, \Omega_{2}^{(0)}$ and $\Omega_{4}^{(0)}$ are provided in Appendix II. Some special cases derived from Eq. 26 are as follows:

\section{Subcase 5.1.1}

When heterogeneity in the layer vanishes, i.e., $\alpha_{1}=0$, Eq. 26 reduces to

$$
\tan \left(k \sqrt{\frac{d_{1}^{\prime} c^{2}}{c_{1}^{2}}-\frac{B_{1}}{B_{5}}}\left(b \cos \Phi x_{1}+h\right)\right)=\frac{p_{r e} \mu_{T}\left(P+R b \Phi \sin \Phi x_{1}\right)}{B_{5} k \sqrt{\frac{d_{1}^{\prime} c^{2}}{c_{1}^{2}}-\frac{B_{1}}{B_{5}}}} .
$$

Equation 27 is the dispersion equation for propagation of $S H$-wave in a homogeneous porous layer, bounded by an upper planar surface and lower corrugated surface, overlying a fibre-reinforced elastic half-space under initial stress.

\section{Subcase 5.1.2}

When the layer is a non-porous solid $(\phi=0)$ and without heterogeneity, i.e., $\alpha_{1}=0, d_{1}^{\prime} \rightarrow 1$, Eq. 26 reduces to

$$
\tan \left(k \sqrt{\frac{c^{2}}{c_{1}^{2}}-\frac{B_{1}}{B_{5}}}\left(b \cos \Phi x_{1}+h\right)\right)=\frac{p_{r e} \mu_{T}\left(P+R b \Phi \sin \Phi x_{1}\right)}{B_{5} k \sqrt{\frac{c^{2}}{c_{1}^{2}}-\frac{B_{1}}{B_{5}}}} .
$$

Equation 28 is the dispersion equation for propagation of $S H$-wave in a homogeneous non-porous layer, bounded by an upper planar surface and lower corrugated surface, overlying a fibre-reinforced elastic half-space under initial stress.

\section{Subcase 5.1.3}

When the layer is isotropic and without heterogeneity, i.e., $\alpha_{1}=0, d_{1}^{\prime} \rightarrow 1$, $B_{1}=B_{5}=\mu_{1}$, Eq. 26 reduces to

$$
\tan \left(k \sqrt{\frac{c^{2}}{c_{1}^{2}}-1}\left(b \cos \Phi x_{1}+h\right)\right)=\frac{p_{r e} \mu_{T}\left(P+R b \Phi \sin \Phi x_{1}\right)}{\mu_{1} k \sqrt{\frac{c^{2}}{c_{1}^{2}}-1}} .
$$

Equation 29 is the dispersion equation for propagation of $S H$-wave in a homogeneous isotropic layer, bounded by an upper planar surface and lower corrugated surface, overlying a fibre-reinforced elastic half-space under initial stress. 


\section{Subcase 5.1.4}

When the layer is isotropic without heterogeneity and half-space is fibrereinforced without initial stress, i.e., $\alpha_{1}=0, d_{1}^{\prime} \rightarrow 1, B_{1}=B_{5}=\mu_{1}, P_{1}=0$, Eq. 26 reduces to

$$
\tan \left(k \sqrt{\frac{c^{2}}{c_{1}^{2}}-1}\left(b \cos \Phi x_{1}+h\right)\right)=\frac{\mu_{T} \sqrt{\frac{Q}{P}-\frac{R^{2}}{4 P^{2}}-\frac{c^{2}}{c_{2}^{2} P}}\left(P+R b \Phi \sin \Phi x_{1}\right)}{\mu_{1} \sqrt{\frac{c^{2}}{c_{1}^{2}}-1}} .
$$

Equation 30 is the dispersion equation for propagation of $S H$-wave in a homogeneous isotropic layer, bounded by an upper planar surface and lower corrugated surface, overlying fibre-reinforced elastic half-space without initial stress.

\section{Subcase 5.1.5}

When the layer is isotropic without heterogeneity and half-space is without initial stress and reinforcement, i.e., $\alpha_{1}=0, d_{1}^{\prime} \rightarrow 1, B_{1}=B_{5}=\mu_{1}, P_{1}=0$, $\mu_{L}=\mu_{T}=\mu_{2}$, Eq. 26 reduces to

$$
\tan \left(k \sqrt{\frac{c^{2}}{c_{1}^{2}}-1}\left(b \cos \Phi x_{1}+h\right)\right)=\frac{\mu_{2} \sqrt{1-\frac{c^{2}}{c_{2}^{2}}}}{\mu_{1} \sqrt{\frac{c^{2}}{c_{1}^{2}}-1}} .
$$

Equation 31 is the dispersion equation for propagation of $S H$-wave in a homogeneous isotropic layer, bounded by an upper planar surface and lower corrugated surface, overlying a half-space without initial stress and reinforcement.

\section{Subcase 5.1.6}

When the layer is isotropic without heterogeneity, half-space is isotropic without initial stress and the lower corrugated boundary surface becomes planar, i.e., $\alpha_{1}=0, d_{1}^{\prime} \rightarrow 1, B_{1}=B_{5}=\mu_{1}, P_{1}=0, \mu_{L}=\mu_{T}=\mu_{2}, b=0$, Eq. 26 becomes (Ewing et al. 1957)

$$
\tan \left(k h \sqrt{\frac{c^{2}}{c_{1}^{2}}-1}\right)=\frac{\mu_{2} \sqrt{1-\frac{c^{2}}{c_{2}^{2}}}}{\mu_{1} \sqrt{\frac{c^{2}}{c_{1}^{2}}-1}}
$$

which is the classical Love wave equation. 


\section{Case 5.2}

When the layer is bounded by an upper corrugated surface, i.e., $x_{3}=a \cos \Phi x_{1}-h$ and a lower planar surface, i.e., $x_{3}=0$ for $\square_{2}=0$, then the dispersion relation 25 reduces to

$$
\tan \left(p_{1}\left(h-a \cos \Phi x_{1}\right)\right)=\frac{\Omega_{1}^{(1)} \Omega_{3}^{(1)}+\Omega_{2}^{(1)} \Omega_{4}^{(1)}}{\left(\Omega_{3}^{(1)}\right)^{2}+\left(\Omega_{4}^{(1)}\right)^{2}},
$$

where $\Omega_{1}^{(1)}, \Omega_{2}^{(1)}, \Omega_{3}^{(1)}$ and $\Omega_{4}^{(1)}$ are provided in Appendix III. Some special cases derived from Eq. 33 are as follows:

\section{Subcase 5.2.1}

When heterogeneity in the layer vanishes, i.e., $\alpha_{1}=0$, Eq. 33 reduces to

$$
\tan \left(k \sqrt{\frac{d_{1}^{\prime} c^{2}}{c_{1}^{2}}-\frac{B_{1}}{B_{5}}}\left(h-a \cos \Phi x_{1}\right)\right)=\frac{\Omega_{1}^{(2)} \Omega_{3}^{(2)}+\Omega_{2}^{(2)} \Omega_{4}^{(2)}}{\left(\Omega_{3}^{(2)}\right)^{2}+\left(\Omega_{4}^{(2)}\right)^{2}},
$$

where $\Omega_{1}^{(2)}, \Omega_{2}^{(2)}, \Omega_{3}^{(2)}$ and $\Omega_{4}^{(2)}$ are provided in Appendix III.

Equation 34 is the dispersion equation for propagation of $S H$-wave in a homogeneous fluid-saturated poroelastic layer, bounded by an upper corrugated surface and lower planar surface, overlying fibre-reinforced half-space under initial stress.

\section{Subcase 5.2.2}

When the layer is a non-porous solid $(\phi=0)$ and without heterogeneity, i.e., $\alpha_{1}=0$, and $d_{1}^{\prime} \rightarrow 1$, Eq. 33 reduces to

$$
\tan \left(k \sqrt{\frac{c^{2}}{c_{1}^{2}}-\frac{B_{1}}{B_{5}}}\left(h-a \cos \Phi x_{1}\right)\right)=\frac{\Omega_{1}^{(3)} \Omega_{3}^{(3)}+\Omega_{2}^{(2)} \Omega_{4}^{(2)}}{\left(\Omega_{3}^{(3)}\right)^{2}+\left(\Omega_{4}^{(2)}\right)^{2}},
$$

where $\Omega_{1}^{(3)}, \Omega_{3}^{(3)}$ are provided in Appendix III.

Equation 35 is the dispersion equation for propagation of $S H$-wave in a homogeneous non-porous layer, bounded by an upper corrugated surface and lower planar surface, overlying a fibre-reinforced half-space under initial stress.

\section{Subcase 5.2.3}

When the layer is isotropic and without heterogeneity, i.e., $\alpha_{1}=0, d_{1}^{\prime} \rightarrow 1$, $B_{1}=B_{5}=\mu_{1}$, Eq. 33 reduces to 


$$
\tan \left(k \sqrt{\frac{c^{2}}{c_{1}^{2}}-1}\left(-a \cos \Phi x_{1}+h\right)\right)=\frac{\Omega_{1}^{(4)} \Omega_{3}^{(4)}+\Omega_{2}^{(3)} \Omega_{4}^{(3)}}{\left(\Omega_{3}^{(4)}\right)^{2}+\left(\Omega_{4}^{(3)}\right)^{2}},
$$

where $\Omega_{1}^{(4)}, \Omega_{2}^{(3)}, \Omega_{3}^{(4)}$ and $\Omega_{4}^{(3)}$ are provided in Appendix III.

Equation 36 is the dispersion equation for propagation of $S H$-wave in a homogeneous isotropic layer, bounded by an upper corrugated surface and lower planar surface, overlying a fibre-reinforced half-space under initial stress.

\section{Subcase 5.2.4}

When the layer is isotropic without heterogeneity and half-space is fibrereinforced without initial stress, i.e., $\alpha_{1}=0$, and $d_{1}^{\prime} \rightarrow 1 \quad B_{1}=B_{5}=\mu_{1}$, $P_{1}=0$, Eq. 33 reduces to

$$
\tan \left(k \sqrt{\frac{c^{2}}{c_{1}^{2}}-1}\left(-a \cos \Phi x_{1}+h\right)\right)=\frac{\Omega_{1}^{(5)} \Omega_{3}^{(4)}+\Omega_{2}^{(3)} \Omega_{4}^{(4)}}{\left(\Omega_{3}^{(4)}\right)^{2}+\left(\Omega_{4}^{(4)}\right)^{2}},
$$

where $\Omega_{1}^{(5)}$ and $\Omega_{4}^{(4)}$ are provided in Appendix III.

Equation 37 is the dispersion equation for propagation of $S H$-wave in a homogeneous isotropic layer, bounded by an upper corrugated surface and lower planar surface, overlying a fibre-reinforced elastic half-space without initial stress.

\section{Subcase 5.2.5}

When the layer is isotropic without heterogeneity and half-space is without initial stress and reinforcement, i.e., $\alpha_{1}=0, d_{1}^{\prime} \rightarrow 1, B_{1}=B_{5}=\mu_{1}, P_{1}=0$, $\mu_{L}=\mu_{T}=\mu_{2}$, Eq. 33 reduces to

$$
\tan \left(k \sqrt{\frac{c^{2}}{c_{1}^{2}}-1}\left(h-a \cos \Phi x_{1}\right)\right)=\frac{-\Omega_{1}^{(6)} \mu_{1}^{2} k^{2}\left(\frac{c^{2}}{c_{1}^{2}}-1\right)-\Omega_{4}^{(5)} k \mu_{1} a \Phi \sin \Phi x_{1}}{\left(\mu_{1}^{2} k^{2}\left(\frac{c^{2}}{c_{1}^{2}}-1\right)\right)^{2}+\left(\Omega_{4}^{(5)}\right)^{2}}
$$

where $\Omega_{1}^{(6)}$ and $\Omega_{4}^{(5)}$ are provided in Appendix III.

Equation 38 is the dispersion equation for propagation of $S H$-wave in homogeneous isotropic layer, bounded by an upper corrugated surface and lower planar surface, overlying a half-space without initial stress and reinforcement. 


\section{Subcase 5.2.6}

When the layer is isotropic without heterogeneity, half-space is reinforcement-free without initial stress and the upper corrugated boundary surface becomes planar, i.e., $\alpha_{1}=0, d_{1}^{\prime} \rightarrow 1, B_{1}=B_{5}=\mu_{1}, P_{1}=0, \mu_{L}=\mu_{T}=\mu_{2}, a=0$, Eq. 33 reduces to Eq. 32, which is the classical Love wave equation (Ewing et al. 1957).

\section{Case 5.3}

When the layer is bounded by an upper corrugated surface, i.e., $x_{3}=a \cos \Phi x_{1}-h$ and a lower corrugated surface, i.e., $x_{3}=b \cos \Phi x_{1}$, then the dispersion relation 25 reduces to

$$
\tan \left(p_{1}\left(h-(a-b) \cos \Phi x_{1}\right)\right)=\frac{\Omega_{1}^{(7)} \Omega_{3}^{(5)}+\Omega_{2}^{(4)} \Omega_{4}^{(6)}}{\left(\Omega_{3}^{(5)}\right)^{2}+\left(\Omega_{4}^{(6)}\right)^{2}} .
$$

where $\Omega_{1}^{(7)}, \Omega_{2}^{(4)}, \Omega_{3}^{(5)}$ and $\Omega_{4}^{(6)}$ are provided in Appendix IV.

Some special cases derived from Eq. 39 are as follows:

\section{Subcase 5.3.1}

When heterogeneity in the layer vanishes, i.e., $\alpha_{1}=0$, Eq. 39 reduces to

$$
\tan \left(k \sqrt{\frac{d_{1}^{\prime} c^{2}}{c_{1}^{2}}-\frac{B_{1}}{B_{5}}}\left(h-(a-b) \cos \Phi x_{1}\right)\right)=\frac{\Omega_{1}^{(8)} \Omega_{3}^{(6)}+\Omega_{2}^{(5)} \Omega_{4}^{(7)}}{\left(\Omega_{3}^{(6)}\right)^{2}+\left(\Omega_{4}^{(7)}\right)^{2}},
$$

where $\Omega_{1}^{(8)}, \Omega_{2}^{(5)}, \Omega_{3}^{(6)}$ and $\Omega_{4}^{(7)}$ are provided in Appendix IV.

Equation 40 is the dispersion equation for propagation of $S H$-wave in a homogeneous fluid-saturated poroelastic layer, bounded by upper and lower corrugated surfaces, overlying a fibre-reinforced elastic half-space under initial stress.

\section{Subcase 5.3.2}

When the layer is a non-porous solid $(\phi=0)$ and without heterogeneity, i.e., $\alpha_{1}=0, d_{1}^{\prime} \rightarrow 1$, Eq. 39 reduces to

$$
\tan \left(k \sqrt{\frac{c^{2}}{c_{1}^{2}}-\frac{B_{1}}{B_{5}}}\left(h-(a-b) \cos \Phi x_{1}\right)\right)=\frac{\Omega_{1}^{(9)} \Omega_{3}^{(7)}+\Omega_{2}^{(5)} \Omega_{4}^{(7)}}{\left(\Omega_{3}^{(7)}\right)^{2}+\left(\Omega_{4}^{(7)}\right)^{2}},
$$

where $\Omega_{1}^{(9)}, \Omega_{3}^{(7)}$ are provided in Appendix IV. 
Equation 41 is the dispersion equation for propagation of $S H$-wave in a homogeneous non-porous layer, bounded by upper and lower corrugated surfaces, overlying a fibre-reinforced elastic half-space under initial stress.

\section{Subcase 5.3.3}

When the layer is isotropic and without heterogeneity, i.e., $\alpha_{1}=0, d_{1}^{\prime} \rightarrow 1$, $B_{1}=B_{5}=\mu_{1}$, Eq. 39 reduces to

$$
\tan \left(k \sqrt{\frac{c^{2}}{c_{1}^{2}}-1}\left(h-(a-b) \cos \Phi x_{1}\right)\right)=\frac{\Omega_{1}^{(10)} \Omega_{3}^{(8)}+\Omega_{2}^{(6)} \Omega_{4}^{(8)}}{\left(\Omega_{3}^{(8)}\right)^{2}+\left(\Omega_{4}^{(8)}\right)^{2}},
$$

where $\Omega_{1}^{(10)}, \Omega_{2}^{(6)}, \Omega_{3}^{(8)}$ and $\Omega_{4}^{(8)}$ are provided in Appendix IV.

Equation 42 is the dispersion equation for propagation of $S H$-wave in a homogeneous isotropic layer, bounded by upper and lower corrugated surfaces, overlying a fibre-reinforced half-space under initial stress.

\section{Subcase 5.3.4}

When the layer is isotropic without heterogeneity and the half-space is fibrereinforced without initial stress, i.e., $\alpha_{1}=0, d_{1}^{\prime} \rightarrow 1, B_{1}=B_{5}=\mu_{1}, P_{1}=0$, Eq. 39 reduces to

$$
\tan \left(k \sqrt{\frac{c^{2}}{c_{1}^{2}}-1}\left(h-(a-b) \cos \Phi x_{1}\right)\right)=\frac{\Omega_{1}^{(11)} \Omega_{3}^{(8)}+\Omega_{2}^{(6)} \Omega_{4}^{(9)}}{\left(\Omega_{3}^{(8)}\right)^{2}+\left(\Omega_{4}^{(9)}\right)^{2}},
$$

where $\Omega_{1}^{(11)}, \Omega_{4}^{(9)}$ are provided in Appendix IV.

Equation 43 is the dispersion equation for propagation of $S H$-wave in a homogeneous isotropic layer, bounded by an upper and lower corrugated surfaces, overlying a fibre-reinforced elastic half-space without initial stress.

\section{Subcase 5.3.5}

When the layer is isotropic without heterogeneity and half-space is isotropic without initial stress and reinforcement, i.e., $\alpha_{1}=0, d_{1}^{\prime} \rightarrow 1 \quad B_{1}=B_{5}=\mu_{1}$, $P_{1}=0, \mu_{L}=\mu_{T}=\mu_{2}$, Eq. 39 reduces to

$$
\tan \left(k \sqrt{\frac{c^{2}}{c_{1}^{2}}-1}\left(h-(a-b) \cos \Phi x_{1}\right)\right)=\frac{\Omega_{1}^{(12)} \Omega_{3}^{(9)}+\Omega_{2}^{(7)} \Omega_{4}^{(10)}}{\left(\Omega_{3}^{(9)}\right)^{2}+\left(\Omega_{4}^{(10)}\right)^{2}},
$$

where $\Omega_{1}^{(12)}, \Omega_{2}^{(7)}, \Omega_{3}^{(9)}$ and $\Omega_{4}^{(10)}$ are provided in Appendix IV. 
Equation 44 is the dispersion equation for propagation of $S H$-wave in a homogeneous isotropic layer, bounded by upper and lower corrugated surfaces, overlying a half-space without initial stress and reinforcement.

\section{Subcase 5.3.6}

When the layer is isotropic without heterogeneity, the half-space is reinforcement-free without initial stress and both the upper and lower corrugated boundary surfaces become planar, i.e., $\alpha_{1}=0, d_{1}^{\prime} \rightarrow 1, B_{1}=B_{5}=\mu_{1}, P_{1}=0$, $\mu_{L}=\mu_{T}=\mu_{2}, a=b=0$, Eq. 39 reduces to Eq. 32, which is the classical Love wave equation (Ewing et al. 1957).

\section{NUMERICAL CALCULATION AND DISCUSSION}

In order to perform numerical computations of the dispersion relation obtained for the propagation of $S H$-wave in a corrugated heterogeneous fluidsaturated poroelastic layer lying over a fibre-reinforced elastic half-space under initial stress, the following data has been taken into consideration:

(a) For the upper corrugated heterogeneous fluid-saturated poroelastic layer (Ke et al. 2006):

$$
B_{1}=3 \times 10^{10} \mathrm{~N} / \mathrm{m}^{2}, B_{5}=4 \times 10^{10} \mathrm{~N} / \mathrm{m}^{2}, \rho_{f}=1000 \mathrm{~kg} / \mathrm{m}^{3} \text { and } \rho_{s}=3000 \mathrm{~kg} / \mathrm{m}^{3} \text {. }
$$

(b) For the lower fibre-reinforced elastic half-space under initial stress (Markham 1970):

$$
\mu_{L}=7.07 \times 10^{10} \mathrm{~N} / \mathrm{m}^{2}, \mu_{T}=3.50 \times 10^{10} \mathrm{~N} / \mathrm{m}^{2} \text { and } \rho_{2}=1600 \mathrm{~kg} / \mathrm{m}^{3} .
$$

In order to perform comparative study of the problem to the case when $\mathrm{SH}$-wave is propagating in a corrugated heterogeneous fluid-saturated poroelastic layer lying over an isotropic (reinforcement-free) half-space under initial stress, the following data has been considered (Gubbins 1990):

(c) For the lower corrugated isotropic half-space (without reinforcement) under initial stress:

$$
\mu_{2}=29.17 \times 10^{10} \mathrm{~N} / \mathrm{m}^{2}, \quad \rho_{2}=5563 \mathrm{~kg} / \mathrm{m}^{3}, a_{1}=a_{3}=0 .
$$

Moreover, the following data has been considered:

$$
\begin{gathered}
\alpha_{1} h=0,0.1,0.2 ; \xi_{1}= \pm 0.1, \pm 0.2, \pm 0.3 ; d_{1}^{\prime}=0.5,0.51,0.52 ; \Phi h=1.1,1.4,1.7 ; \\
k h=5, a \Phi=0.1,0.15,0.2,0.3,0.5 ; b \Phi=0.1,0.15,0.2,0.3,0.5 ; \\
x_{1} / h=0.01,0.05,0.09 ; a_{1}=0.00316227 ; \phi=0.2 .
\end{gathered}
$$

The effect of heterogeneity and porosity present in the upper layer, initial stress acting in the lower fibre-reinforced elastic half-space, corrugation parameters associated with the upper and lower corrugated boundary surfaces 
of the layer, undulatory parameters and position parameter on the phase velocity has been demonstrated in Figs. 2-7. Moreover, with a view to demonstrate the effect of presence and absence of reinforcement on the phase velocity, curves have been plotted after carrying out numerical computation. In each of the figures, curves 1,2 , and 3 correspond to the case when corrugated heterogeneous fluid-saturated poroelastic layer lies over a fibrereinforced elastic half-space under initial stress and curves 4, 5, and 6 are associated to the case when corrugated heterogeneous fluid-saturated poroelastic layer lies over a reinforcement-free half-space under initial stress.
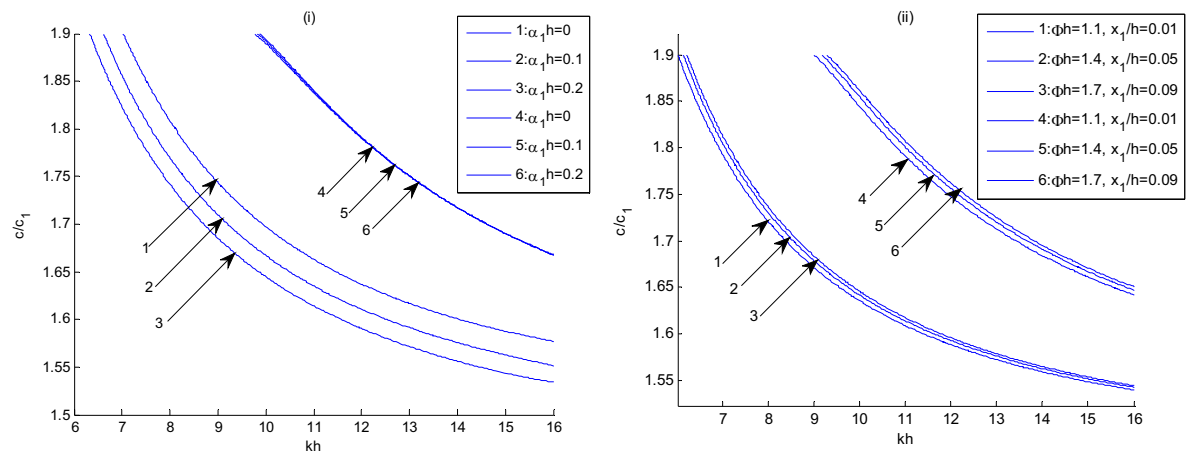

Fig. 2. Variation of phase velocity $\left(c / c_{1}\right)$ against wave number $(k h)$ : (i) for different values of heterogeneity parameter $\left(\alpha_{1} h\right)$ of the layer when $\Phi h=1.4, a \Phi=0.1$, and $x_{1} / h=0.05$; (ii) for different values of undulatory parameter $(\Phi h)$ along with different values of position parameter $\left(x_{1} / h\right)$ when $\alpha_{1} h=0.1$ and $a \Phi=0$ (Case 5.1); for fixed $d_{1}^{\prime}=0.5, \xi_{1}=0.1, b \Phi=0.1$.
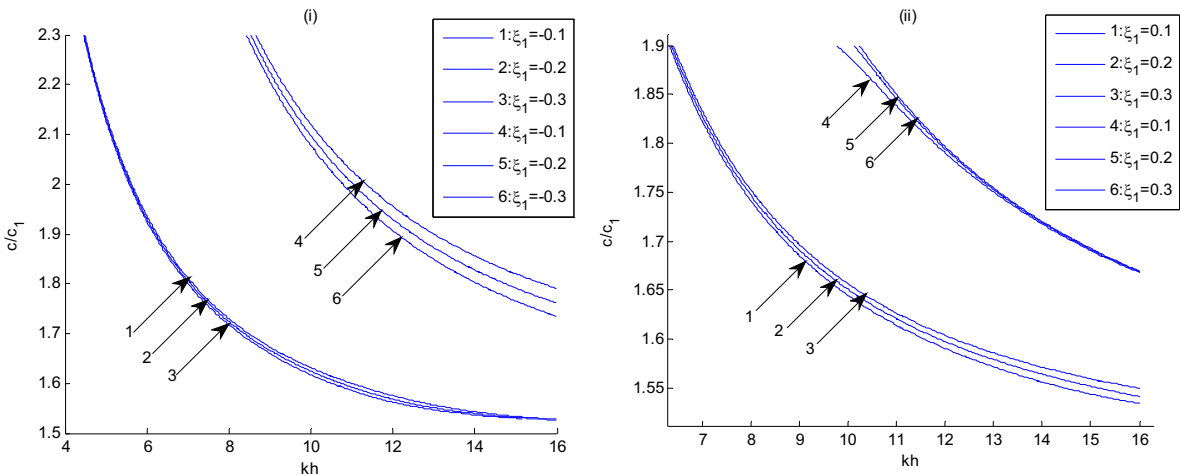

Fig. 3. Variation of phase velocity $\left(c / c_{1}\right)$ against wave number $(k h)$ : (i) for different values of horizontal tensile initial stress acting in the half-space $\left(\xi_{1}\right)$ and (ii) for different values of horizontal compressive initial stress acting in the half-space $\left(\xi_{1}\right)$; for fixed $d_{1}^{\prime}=0.5, \alpha_{1} h=0.1, \Phi h=1.4, a \Phi=0.1, b \Phi=0.1, x_{1} / h=0.05$. 
(i)

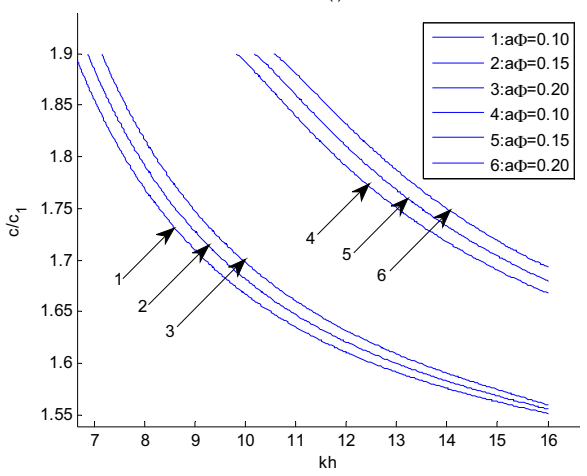

(ii)

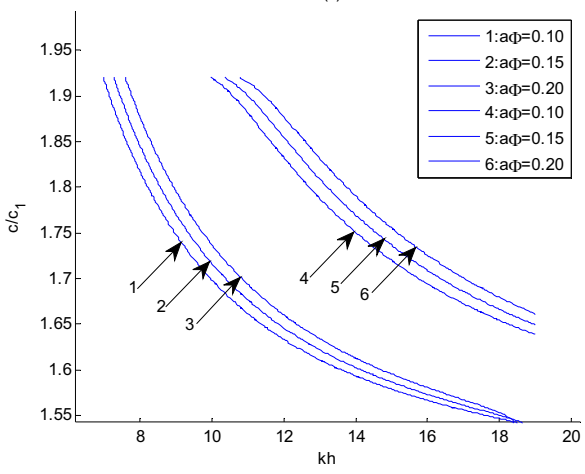

Fig. 4. Variation of phase velocity $\left(c / c_{1}\right)$ against wave number $(k h)$ : (i) for different values of corrugation parameter associated with the upper boundary surface of layer $(a \Phi)$ when $b \Phi=0.1$ (Case 5.3) and (ii) for different values of corrugation parameter of upper boundary surface of layer $(a \Phi)$ when $b \Phi=0$ (Case 5.2) for fixed $d_{1}^{\prime}=0.5$, $\xi_{1}=0.1, a_{1} / h=0.1, \Phi h=1.4, x_{1} / h=0.05$.

(i)

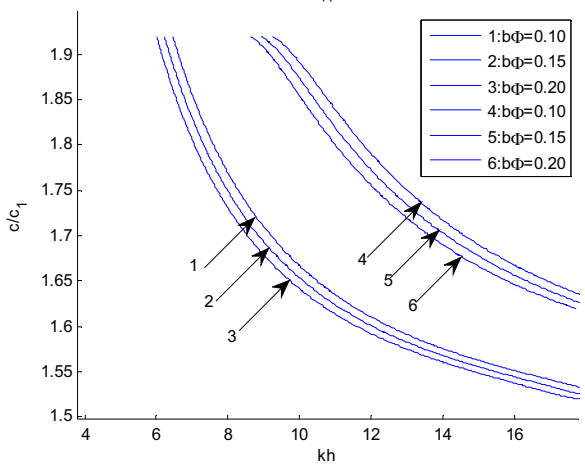

(ii)

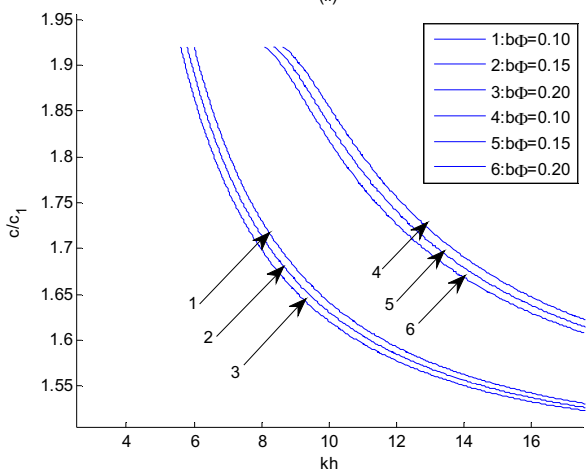

Fig. 5. Variation of phase velocity $\left(c / c_{1}\right)$ against wave number $(k h)$ : (i) for different values of corrugation parameter associated with the interface between layer and halfspace $(b \Phi)$ when $a \Phi=0.1$ (Case 5.3), and (ii) for different values of corrugation parameter associated with the interface between layer and half-space $(b \Phi)$ when $a \Phi=0$ (Case 5.3), for fixed $d_{1}^{\prime}=0.5, \xi_{1}=0.1, \alpha_{1} h=0.1, \Phi h=1.4, x_{1} / h=0.05$.

The variation of phase velocity $\left(c / c_{1}\right)$ against wave number $(k h)$ has been illustrated in Figs. 2-5. These figures show that the phase velocity of $\mathrm{SH}$ wave decreases with increasing wave number. Figures $2 \mathrm{i}$-ii represent the effect of variation of heterogeneity parameter of the layer and undulatory parameter along with position parameter, respectively, on the phase velocity of $S H$-wave. These figures indicate that phase velocity of $S H$-wave decreases with increasing heterogeneity parameter whereas it increases with increasing 


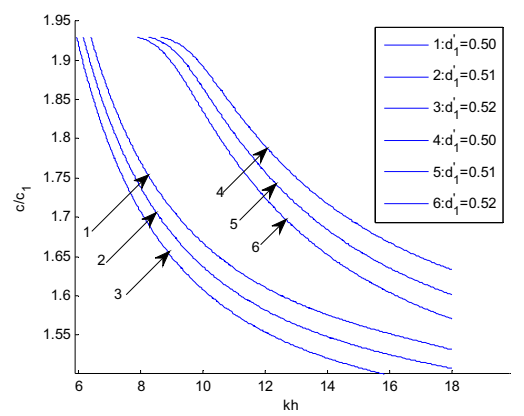

Fig. 6. Variation of phase velocity $\left(c / c_{1}\right)$ against wave number $(k h)$ for different values of porosity of layer $\left(d_{1}^{\prime}\right)$ for fixed $\xi_{1}=0.1, \alpha_{1} h=0.1, a \Phi=0.1, b \Phi=0.1$, $x_{1} / h=0.05, \Phi h=1.4$.
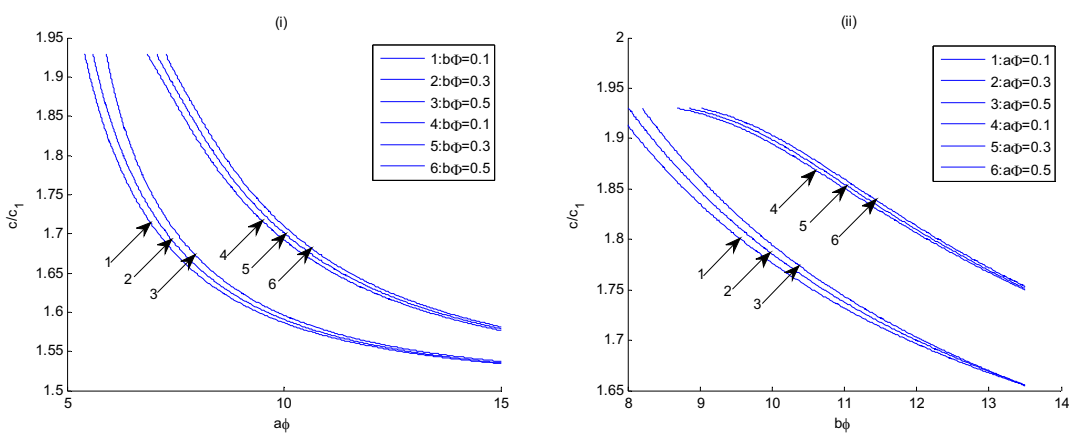

Fig. 7. Variation of phase velocity $\left(c / c_{1}\right)$ against: (i) corrugation parameter of upper boundary surface of layer $(a \Phi)$ for different values of corrugation parameter of lower boundary surface of layer $(b \Phi)$, and (ii) corrugation parameter of lower boundary surface of layer $(b \Phi)$ for different values of corrugation parameter of upper boundary surface of layer $(a \Phi)$, for fixed $\alpha_{1} h=0.1, \xi_{1}=0.1, d_{1}^{\prime}=0.5, x_{1} / h=0.05, k h=5$, $\Phi h=1.4$.

undulatory parameter along with position parameter for both cases when the lower half-space is reinforced or reinforcement-free. Moreover, it has been realized through comparative study that the influence of heterogeneity of layer on phase velocity for the case when the elastic half-space is with reinforcement is much more pronounced as compared to the case when the elastic half-space is without reinforcement. Further, it could be quoted from Fig. 2(ii) that the phase velocity of $S H$-wave will be greater if corrugated boundary surfaces of the layer are steeply undulated and phase velocity of $S H$-wave will be lesser if corrugated boundary surfaces of the layer are modestly undulated for both cases when the lower half-space is with reinforce- 
ment and without reinforcement. Curve 1 in Fig. 2(i) corresponds to Subcase 5.3.1 and the curves plotted in Fig. 2(ii) correspond to Case 5.1.

The effect of horizontal tensile initial stress and horizontal compressive initial stress acting in the lower half-space, on phase velocity of $S H$-wave, has been represented in Figs. 3(i) and 3(ii), respectively. It is observed from these figures that the phase velocity decreases with increasing horizontal tensile initial stress and phase velocity increases with increasing horizontal compressive initial stress. Meticulous examination of Figs. 3(i) and 3(ii) concludes that the effect of horizontal tensile initial stress on phase velocity is greater for the case when the lower half-space is without reinforcement, whereas the effect of horizontal compressive initial stress on phase velocity is greater for the case when the lower half-space is under reinforcement.

Figures 4(i) and 4(ii) depicture the effect of corrugation parameter of the uppermost free boundary surface of layer $(a \Phi)$ on the phase velocity when the common interface of the layer and half-space $(b \Phi)$ is considered corrugated and planar, respectively. Curves plotted in Fig. 4(i) correspond to Case 5.3 and curves plotted in Fig. 4(ii) correspond to Case 5.2. These figures establish that phase velocity of $\mathrm{SH}$-wave increases with increasing corrugation parameter associated with uppermost boundary surface of the layer for both cases when the lower elastic half-space is with reinforcement and without reinforcement. Also, it has been noted that the dispersion curves vary significantly with a slight change in corrugation parameter of uppermost free boundary surface of the layer.

Figures 5(i) and 5(ii) demonstrate the effect of corrugation parameter associated with the common interface of the layer and half-space $(b \Phi)$ on phase velocity for the case when the corrugation parameter of the upper free boundary surface of the layer is corrugated and planar, respectively. Figure $5 \mathrm{i}$ is associated to Case 5.3 and Fig. 5(ii) corresponds to Case 5.1. These figures suggest that phase velocity of $\mathrm{SH}$-wave decreases with increasing corrugation parameter associated with the common interface of the layer and half space for both cases when the lower elastic half-space is reinforced and reinforcement-free. More interestingly, it has been observed that for a slight change in corrugation parameter associated with the common interface of the layer and half-space, the dispersion curve is affected considerably.

Curves plotted in Fig. 6 set forth the influence of porosity of the layer $\left(d_{1}^{\prime}\right)$ on phase velocity of $S H$-wave. It could be notified from the figure that phase velocity decreases with increasing porosity of the layer for both cases when the lower elastic half-space is with reinforcement and without reinforcement. Moreover, it could be added that porosity of the upper layer has an immense effect on the phase velocity as a slight increase in porosity of layer causes a significant decrement in phase velocity of $\mathrm{SH}$-wave. 
The variation of phase velocity against corrugation parameter of the uppermost stress free boundary surface of the layer has been carved out in Fig. 7(i) for different values of corrugation parameter of the lower boundary surface of the layer. Phase velocity is found to be decreasing with increase in corrugation parameter of the uppermost free boundary surface of the layer for both cases when the lower half-space is reinforced and reinforcementfree. Moreover, it has been observed from Fig. 7(i) that with increase in corrugation parameter of the lower boundary surface of layer, phase velocity increases. Figure 7(ii) shows the variation of phase velocity against corrugation parameter of the lower boundary surface of layer for different values of corrugation parameter of the uppermost free boundary surface of layer. This figure concludes that phase velocity of $S H$-wave decreases with increasing corrugation parameter of the lower boundary surface of layer. Figure 7(ii) establishes that for both cases, when the lower half-space is with reinforcement and without reinforcement, there is a monotonic increase in phase velocity with increasing corrugation parameter of the uppermost stress-free boundary surface. Meticulous examination of Fig. 7(ii) shows that the effect of corrugation parameter of the uppermost stress-free boundary surface on phase velocity is more pronounced for the case when the lower half-space reinforced than the case when the lower half-space is reinforcement-free.

In general, all the figures elucidate that the dispersion curves corresponding to the case when the half-space is without reinforcement are lying much above to the curves associated with case when the half-space is with reinforcement. This implies that reinforcement present in the half-space disfavours the phase velocity of $S H$-wave. Therefore, comparative study made in all the figures for reinforced and reinforcement-free case of elastic half-space manifest that as reinforcement prevails in semi-infinite medium, phase velocity of $\mathrm{SH}$-wave gets decreased.

\section{CONCLUSIONS}

The present paper reports the study of propagation of $\mathrm{SH}$-wave in a corrugated heterogeneous fluid-saturated poroelastic layer lying over an initially stressed fibre-reinforced elastic half-space. The dispersion relation has been found in closed form. The effect of the corrugation parameter associated with the upper and lower boundary surfaces of the layer, heterogeneity, initial stress, porosity, reinforcement, undulation parameter and position parameter on the phase velocity has been studied in comparative manner and demonstrated by means of graphs through numerical computation. The prime outcomes of the study may be concluded as follows: 
- Phase velocity of $S H$-wave decreases with increasing wave number for both cases, when the lower half-space is reinforced and reinforcementfree.

- Dispersion curves corresponding to the case when the half-space is without reinforcement lies above to the dispersion curves associated with case when the half-space is with reinforcement. This establishes that phase velocity is favoured by the situation when the reinforcement prevails in semi-infinite elastic medium.

- The corrugation parameter of upper and lower boundary surfaces of the layer has contrary effect on the phase velocity of $\mathrm{SH}$-wave; as the phase velocity increases with increasing corrugation parameter of the uppermost stress-free boundary surface of the layer, whereas it decreases with increasing the corrugation parameter of the lower boundary surface of the layer for both cases, when the lower half-space is with reinforcement and without reinforcement.

a The phase velocity of $S H$-wave is greater for the case when corrugated boundary surfaces of the layer are steeply undulated as compared to the case when corrugated boundary surfaces of the layer are modestly undulated, regardless of the fact whether the lower elastic half-space is with reinforcement or without reinforcement.

- The undulatory parameter and horizontal compressive initial stress acting in elastic half-space has a favouring effect on phase velocity, i.e., phase velocity increases with increasing undulatory parameter and horizontal compressive initial stress acting in elastic half-space.

- The heterogeneity of layer and porosity, and horizontal tensile initial stress acting in elastic half-space has a disfavouring effect on phase velocity, i.e., phase velocity decreases with increasing heterogeneity and porosity of the layer, and horizontal tensile initial stress acting in elastic half-space.

- The heterogeneity of the layer has a much pronounced effect on phase velocity for the case when the lower half-space is reinforced compared to the case when lower half-space is reinforcement-free.

- In the absence of heterogeneity and porosity in the layer, initial stress and reinforcement in the half-space obtained dispersion relation reduces to the classical Love wave equation, if the corrugated boundary surface of the layer becomes planar. This provides validation of the present problem.

Acknowledgements. The authors convey their sincere thanks to Indian Institute of Technology (Indian School of Mines), Dhanbad, for facilitating us with its best facility for research. 


\section{Appendix I}

$$
\begin{aligned}
T_{1}= & \left(\frac{\alpha_{1}}{2} \tilde{B}_{5}+i k \Upsilon_{1}^{\prime} \tilde{B}_{1}\right), T_{2}=\tilde{B}_{5} p_{1}, T_{3}=\left(\frac{\alpha_{1}}{2} \tilde{B}_{5}+i k \Upsilon_{2}^{\prime} \tilde{B}_{1}\right), \\
T_{4}= & p_{2} \mu_{T}\left(P-\Upsilon_{2}^{\prime} R\right)-i k \mu_{T}\left(R-\Upsilon_{2}^{\prime} Q\right), \\
\Omega_{1}= & \tilde{B}_{5} p_{1}\left(\alpha_{1} \tilde{B}_{5}-p_{r e} \mu_{T} e^{-\alpha_{1}\left(\Upsilon_{2}+h\right)}\left(P-\Upsilon_{2}^{\prime} R\right)\right), \\
\Omega_{2}= & k \tilde{B}_{1}\left(\Upsilon_{2}^{\prime}+\Upsilon_{1}^{\prime}\right)+\mu_{T}\left(k\left(R-\Upsilon_{2}^{\prime} Q\right)-p_{i m} e^{-\alpha_{1}\left(\Upsilon_{2}+h\right)}\left(P-\Upsilon_{2}^{\prime} R\right)\right), \\
\Omega_{3}= & e^{-\alpha_{1}\left(\Upsilon_{2}+h\right)}\left\{\mu_{T}\left(P-\Upsilon_{2}^{\prime} R\right)\left(\frac{\alpha_{1}}{2} \tilde{B}_{5} p_{r e}-k \Upsilon_{1}^{\prime} \tilde{B}_{1} p_{i m}\right)+\tilde{B}_{1} \Upsilon_{1}^{\prime} k^{2} \mu_{T}\left(R-\Upsilon_{2}^{\prime} Q\right)\right\} \\
& -\tilde{B}_{5}^{2}\left(p_{1}^{2}+\frac{\alpha_{1}^{2}}{4}\right)+k^{2} \tilde{B}_{1}^{2} \Upsilon_{2}^{\prime} \Upsilon_{1}^{\prime}, \\
\Omega_{4}= & e^{-\alpha_{1}\left(\Upsilon_{2}+h\right)} \mu_{T}\left\{\left(P-\Upsilon_{2}^{\prime} R\right)\left(\frac{\alpha_{1}}{2} \tilde{B}_{5} p_{i m}+k \Upsilon_{1}^{\prime} \tilde{B}_{1} p_{r e}\right)-\frac{\alpha_{1}}{2} \tilde{B}_{5} k\left(R-\Upsilon_{2}^{\prime} Q\right)\right\} \\
& -\frac{\alpha_{1}}{2} \tilde{B}_{5} \tilde{B}_{1} k\left(\Upsilon_{2}^{\prime}+\Upsilon_{1}^{\prime}\right),
\end{aligned}
$$

\section{Appendix II}

$\Omega_{1}^{(0)}=\tilde{B}_{5} p_{1}\left(\alpha_{1} \tilde{B}_{5}-p_{r e} \mu_{T}\left(P+R b \Phi \sin \Phi x_{1}\right) e^{-\alpha_{1}\left(b \cos \Phi x_{1}+h\right)}\right)$,

$\Omega_{2}^{(0)}=-k \tilde{B}_{1} b \Phi \sin \Phi x_{1}+\left(k \mu_{T}\left(R+Q b \Phi \sin \Phi x_{1}\right)-p_{i m} \mu_{T}\left(P+R b \Phi \sin \Phi x_{1}\right) e^{-\alpha_{1}\left(b \cos \Phi x_{1}+h\right)}\right)$,

$\Omega_{3}^{(0)}=e^{-\alpha_{1}\left(b \cos \Phi x_{1}+h\right)}\left\{\mu_{T}\left(P+R b \Phi \sin \Phi x_{1}\right) \frac{\alpha_{1}}{2} \tilde{B}_{5} p_{r e}\right\}-\tilde{B}_{5}^{2}\left(p_{1}^{2}+\frac{\alpha_{1}^{2}}{4}\right)$,

$\Omega_{4}^{(0)}=\frac{e^{-\alpha_{1}\left(b \cos \Phi x_{1}+h\right)}}{2}\left\{\mu_{T}\left(P+R b \Phi \sin \Phi x_{1}\right) \alpha_{1} \tilde{B}_{5} p_{i m}-\alpha_{1} \tilde{B}_{5} k \mu_{T} \times\right.$

$\left.\times\left(R+Q b \Phi \sin \Phi x_{1}\right)\right\}+\frac{\alpha_{1}}{2} \tilde{B}_{5} \tilde{B}_{1} k b \Phi \sin \Phi x_{1}$,

\section{Appendix III}

$$
\begin{aligned}
& \Omega_{1}^{(1)}=\tilde{B}_{5} p_{1}\left(\alpha_{1} \tilde{B}_{5}-p_{r e} \mu_{T} P e^{-\alpha_{1} h}\right), \Omega_{2}^{(1)}=-k \tilde{B}_{1} a \Phi \sin \Phi x_{1}+\left(k \mu_{T} R-p_{i m} \mu_{T} P e^{-\alpha_{1} h}\right), \\
& \Omega_{3}^{(1)}=e^{-\alpha_{1} h} \mu_{T}\left\{P\left(\frac{\alpha_{1}}{2} \tilde{B}_{5} p_{r e}+k \tilde{B}_{1} p_{i m} a \Phi \sin \Phi x_{1}\right)-\tilde{B}_{1} k^{2} R a \Phi \sin \Phi x_{1}\right\}-\tilde{B}_{5}^{2}\left(p_{1}^{2}+\frac{\alpha_{1}^{2}}{4}\right),
\end{aligned}
$$


$\Omega_{4}^{(1)}=e^{-\alpha_{1} h} \mu_{T}\left\{P\left(\frac{\alpha_{1}}{2} \tilde{B}_{5} p_{i m}-k \tilde{B}_{1} p_{r e} a \Phi \sin \Phi x_{1}\right)-\frac{\alpha_{1}}{2} \tilde{B}_{5} k R\right\}+\frac{\alpha_{1}}{2} \tilde{B}_{5} \tilde{B}_{1} k a \Phi \sin \Phi x_{1}$,

$\Omega_{1}^{(2)}=-p_{r e} \mu_{T} k P B_{5} \sqrt{\frac{d_{1}^{\prime} c^{2}}{c_{1}^{2}}-\frac{B_{1}}{B_{5}}}, \Omega_{2}^{(2)}=-k B_{1} a \Phi \sin \Phi x_{1}+k \mu_{T} R-p_{i m} \mu_{T} P$,

$\Omega_{3}^{(2)}=\mu_{T} P k B_{1} p_{i m} a \Phi \sin \Phi x_{1}-B_{1} k^{2} \mu_{T} R a \Phi \sin \Phi x_{1}-B_{5}^{2} k^{2}\left(\frac{d_{1}^{\prime} c^{2}}{c_{1}^{2}}-\frac{B_{1}}{B_{5}}\right)$,

$\Omega_{4}^{(2)}=-\mu_{T} P k B_{1} p_{r e} a \Phi \sin \Phi x_{1}, \quad \Omega_{1}^{(3)}=-p_{r e} \mu_{T} P B_{5} k \sqrt{\frac{c^{2}}{c_{1}^{2}}-\frac{B_{1}}{B_{5}}}$,

$\Omega_{3}^{(3)}=\mu_{T} P k B_{1} p_{i m} a \Phi \sin \Phi x_{1}-B_{1} k^{2} \mu_{T} R a \Phi \sin \Phi x_{1}-B_{5}^{2} k^{2}\left(\frac{c^{2}}{c_{1}^{2}}-\frac{B_{1}}{B_{5}}\right)$,

$\Omega_{1}^{(4)}=-p_{r e} \mu_{T} P \mu_{1} k \sqrt{\frac{c^{2}}{c_{1}^{2}}-1}, \Omega_{2}^{(3)}=-k \mu_{1} a \Phi \sin \Phi x_{1}+\frac{k R \mu_{T}}{2}$,

$\Omega_{3}^{(4)}=\mu_{T} k \mu_{1} \frac{k R}{2} a \Phi \sin \Phi x_{1}-\mu_{1} k^{2} \mu_{T} R a \Phi \sin \Phi x_{1}-\mu_{1}^{2} k^{2}\left(\frac{c^{2}}{c_{1}^{2}}-1\right)$,

$\Omega_{4}^{(3)}=-\mu_{T} P k \mu_{1} p_{r e} a \Phi \sin \Phi x_{1}, \Omega_{4}^{(4)}=-k \mu_{T} P \mu_{1} k \sqrt{\frac{Q}{P}-\frac{R^{2}}{4 P^{2}}-\frac{c^{2}}{c_{2}^{2} P}} a \Phi \sin \Phi x_{1}$,

$\Omega_{1}^{(5)}=-\mu_{T} P k^{2} \mu_{1} \sqrt{\frac{c^{2}}{c_{1}^{2}}-1} \sqrt{\frac{Q}{P}-\frac{R^{2}}{4 P^{2}}-\frac{c^{2}}{c_{2}^{2} P}}$,

$\Omega_{1}^{(6)}=-\mu_{2} \mu_{1} k^{2} \sqrt{1-\frac{c^{2}}{c_{2}^{2}}} \sqrt{\frac{c^{2}}{c_{1}^{2}}-1}, \quad \Omega_{4}^{(5)}=-\mu_{2} P \mu_{1} k^{2} \sqrt{1-\frac{c^{2}}{c_{2}^{2}}} a \Phi \sin \Phi x_{1}$,

\section{Appendix IV}

$$
\begin{aligned}
\Omega_{1}^{(7)} & =\tilde{B}_{5} p_{1}\left(\alpha_{1} \tilde{B}_{5}-p_{r e} \mu_{T}\left(P+R b \Phi \sin \Phi x_{1}\right) e^{-\alpha_{1}\left(b \cos \Phi x_{1}+h\right)}\right), \\
\Omega_{2}^{(4)} & =-k \tilde{B}_{1}(b+a) \Phi \sin \Phi x_{1}+\left(k \mu_{T}\left(R+Q b \Phi \sin \Phi x_{1}\right)\right. \\
& \left.-p_{i m} \mu_{T}\left(P+R b \Phi \sin \Phi x_{1}\right) e^{-\alpha_{1}\left(b \cos \Phi x_{1}+h\right)}\right),
\end{aligned}
$$

$\Omega_{3}^{(5)}=e^{-\alpha_{1}\left(b \cos \Phi x_{1}+h\right)}\left\{\mu_{T}\left(P+R b \Phi \sin \Phi x_{1}\right)\left(\frac{\alpha_{1}}{2} \tilde{B}_{5} p_{r e}+k \tilde{B}_{1} p_{i m} a \Phi \sin \Phi x_{1}\right)-\right.$

$\left.-\tilde{B}_{1} k^{2} \mu_{T}\left(R+Q b \Phi \sin \Phi x_{1}\right) a \Phi \sin \Phi x_{1}\right\}-\tilde{B}_{5}^{2}\left(p_{1}^{2}+\frac{\alpha_{1}^{2}}{4}\right)+k^{2} \tilde{B}_{1}^{2} a b \Phi^{2}\left(\sin \Phi x_{1}\right)^{2}$, 


$$
\begin{aligned}
\Omega_{4}^{(6)}= & e^{-\alpha_{1}\left(b \cos \Phi x_{1}+h\right)}\left\{\mu_{T}\left(P+R b \Phi \sin \Phi x_{1}\right)\left(\frac{\alpha_{1}}{2} \tilde{B}_{5} p_{i m}+k \tilde{B}_{1} p_{r e} a \Phi \sin \Phi x_{1}\right)-\right. \\
& \left.-\frac{\alpha_{1}}{2} \tilde{B}_{5} k \mu_{T}\left(R+Q b \Phi \sin \Phi x_{1}\right)\right\}+\frac{\alpha_{1}}{2} \tilde{B}_{5} \tilde{B}_{1} k(b+a) \Phi \sin \Phi x_{1},
\end{aligned}
$$

$\Omega_{1}^{(8)}=-p_{r e} \mu_{T} B_{5} k \sqrt{\frac{d_{1}^{\prime} c^{2}}{c_{1}^{2}}-\frac{B_{1}}{B_{5}}}\left(P+R b \Phi \sin \Phi x_{1}\right)$,

$\Omega_{2}^{(5)}=-k B_{1}(b+a) \Phi \sin \Phi x_{1}+k \mu_{T}\left(R+Q b \Phi \sin \Phi x_{1}\right)-p_{i m} \mu_{T}\left(P+R b \Phi \sin \Phi x_{1}\right)$, $\Omega_{3}^{(6)}=\mu_{T}\left(P+R b \Phi \sin \Phi x_{1}\right)\left(k B_{1} p_{\text {im }} a \Phi \sin \Phi x_{1}\right)-B_{1} k^{2} \mu_{T}\left(R+Q b \Phi \sin \Phi x_{1}\right) \times$ $\times a \Phi \sin \Phi x_{1}-B_{5}^{2} k^{2}\left(\frac{d_{1}^{\prime} c^{2}}{c_{1}^{2}}-\frac{B_{1}}{B_{5}}\right)+k^{2} B_{1}^{2} a b \Phi^{2}\left(\sin \Phi x_{1}\right)^{2}$,

$\Omega_{4}^{(7)}=\mu_{T}\left(P+R b \Phi \sin \Phi x_{1}\right)\left(k B_{1} p_{r e} a \Phi \sin \Phi x_{1}\right)$,

$\Omega_{1}^{(9)}=-p_{r e} \mu_{T} B_{5} k \sqrt{\frac{c^{2}}{c_{1}^{2}}-\frac{B_{1}}{B_{5}}}\left(P+R b \Phi \sin \Phi x_{1}\right)$,

$\Omega_{2}^{(5)}=-k B_{1}(b+a) \Phi \sin \Phi x_{1}+k \mu_{T}\left(R+Q b \Phi \sin \Phi x_{1}\right)-p_{i m} \mu_{T}\left(P+R b \Phi \sin \Phi x_{1}\right)$,

$\Omega_{3}^{(7)}=\mu_{T}\left(P+R b \Phi \sin \Phi x_{1}\right) k B_{1} p_{i m} a \Phi \sin \Phi x_{1}-B_{1} k^{2} \mu_{T}\left(R+Q b \Phi \sin \Phi x_{1}\right) \times$ $\times a \Phi \sin \Phi x_{1}-B_{5}^{2} k^{2}\left(\frac{c^{2}}{c_{1}^{2}}-\frac{B_{1}}{B_{5}}\right)+k^{2} B_{1}^{2} a b \Phi^{2}\left(\sin \Phi x_{1}\right)^{2}$,

$\Omega_{1}^{(10)}=-p_{r e} \mu_{T} \mu_{1} k \sqrt{\frac{c^{2}}{c_{1}^{2}}-1}\left(P+R b \Phi \sin \Phi x_{1}\right)$,

$\Omega_{2}^{(6)}=-k \mu_{1}(b+a) \Phi \sin \Phi x_{1}+k \mu_{T}\left(R+Q b \Phi \sin \Phi x_{1}\right)-p_{i m} \mu_{T}\left(P+R b \Phi \sin \Phi x_{1}\right)$,

$\Omega_{3}^{(8)}=\mu_{T} k \mu_{1} p_{i m}\left(P+R b \Phi \sin \Phi x_{1}\right) a \Phi \sin \Phi x_{1}-\mu_{1} k^{2} \mu_{T}\left(R+Q b \Phi \sin \Phi x_{1}\right) \times$ $\times a \Phi \sin \Phi x_{1}-\mu_{1}^{2} k^{2}\left(\frac{c^{2}}{c_{1}^{2}}-1\right)+k^{2} \mu_{1}^{2} a b \Phi^{2}\left(\sin \Phi x_{1}\right)^{2}$,

$\Omega_{4}^{(8)}=\mu_{T} k \mu_{1} p_{r e} a \Phi \sin \Phi x_{1}\left(P+R b \Phi \sin \Phi x_{1}\right)$,

$\Omega_{1}^{(11)}=-\mu_{1} k^{2} \mu_{T}\left(P+R b \Phi \sin \Phi x_{1}\right) \sqrt{\frac{c^{2}}{c_{1}^{2}}-1} \sqrt{\frac{Q}{P}-\frac{R^{2}}{4 P^{2}}-\frac{c^{2}}{c_{2}^{2} P}}$,

$\Omega_{2}^{(6)}=-k \mu_{1}(b+a) \Phi \sin \Phi x_{1}+k \mu_{T}\left(R+Q b \Phi \sin \Phi x_{1}\right)-\frac{\mu_{T} k R}{2 P}\left(P+R b \Phi \sin \Phi x_{1}\right)$, 


$$
\begin{aligned}
\Omega_{3}^{(8)} & =\frac{\mu_{T} \mu_{1} k^{2} R}{2 P} a \Phi \sin \Phi x_{1}\left(P+R b \Phi \sin \Phi x_{1}\right)-\mu_{1} k^{2} \mu_{T}\left(R+Q b \Phi \sin \Phi x_{1}\right) \times \\
& \times a \Phi \sin \Phi x_{1}-\mu_{1}^{2} k^{2}\left(\frac{c^{2}}{c_{1}^{2}}-1\right)+k^{2} \mu_{1}^{2} a b \Phi^{2}\left(\sin \Phi x_{1}\right)^{2}
\end{aligned}
$$

$\Omega_{4}^{(9)}=\mu_{T} \mu_{1} k^{2}\left(P+R b \Phi \sin \Phi x_{1}\right) \sqrt{\frac{Q}{P}-\frac{R^{2}}{4 P^{2}}-\frac{c^{2}}{c_{2}^{2} P}} a \Phi \sin \Phi x_{1}$,

$\Omega_{1}^{(12)}=-\mu_{1} \mu_{T} k^{2} \sqrt{\frac{c^{2}}{c_{1}^{2}}-1} \sqrt{1-\frac{c^{2}}{c_{2}^{2}}}, \Omega_{4}^{(10)}=k \mu_{T} \mu_{1} k \sqrt{1-\frac{c^{2}}{c_{2}^{2}}} a \Phi \sin \Phi x_{1}$,

$\Omega_{2}^{(7)}=-k \mu_{1}(b+a) \Phi \sin \Phi x_{1}+k \mu_{T} b \Phi \sin \Phi x_{1}$,

$\Omega_{3}^{(9)}=\left(\mu_{1}-\mu_{T}\right) \mu_{1} k^{2} a b \Phi^{2}\left(\sin \Phi x_{1}\right)^{2}-\mu_{1}^{2} k^{2}\left(\frac{c^{2}}{c_{1}^{2}}-1\right)$.

\section{References}

Asano, S. (1966), Reflection and refraction of elastic waves at a corrugated interface, Bull. Seismol. Soc. Am. 56, 1, 201-222.

Belfield, A.J., T.G. Rogers, and A.J.M. Spencer (1983), Stress in elastic plates reinforced by fibres lying in concentric circles, J. Mech. Phys. Solids 31, 1, 2554, DOI: 10.1016/0022-5096(83)90018-2.

Biot, M.A. (1962), Mechanics of deformation and acoustic propagation in porous media, J. Appl. Phys. 33, 4, 1482-1490, DOI: 10.1063/1.1728759.

Biot, M.A. (1965), Mechanics of Incremental Deformations, Wiley, New York.

Chattaraj, R., S.K. Samal, and N.C. Mahanti (2013), Dispersion of Love wave propagating in irregular anisotropic porous stratum under initial stress, Int. J. Geomech. 13, 4, 402-408, DOI: 10.1061/(ASCE)GM.1943-5622. 0000230 .

Chattopadhyay, A., and S. Choudhury (1990), Propagation, reflection and transmission of magneto- elastic shear waves in a self-reinforced medium, Int. J. Eng. Sci. 28, 6, 485-495, DOI: 10.1016/0020-7225(90)90051-J.

Chattopadhyay, A., and S. Choudhury (1995), Magnetoelastic shear waves in an infinite self-reinforced plate, Int. J. Num. Analytical Meth. Geomech. 19, 4, 289-304, DOI: 10.1002/nag.1610190405. 
Chattopadhyay, A., and A.K. Singh (2012a), G-type seismic waves in fibre reinforced media, Meccanica 47, 7, 1775-1785, DOI: 10.1007/s11012-0129553-2.

Chattopadhyay, A., and A.K. Singh (2012b), Propagation of magnetoelastic shear waves in an irregular self-reinforced layer, J. Eng. Math. 75, 1, 139-155, DOI: $10.1007 / \mathrm{s} 10665-011-9519-8$.

Chattopadhyay, A., S. Gupta, A.K. Singh, and S.A. Sahu (2010a), Propagation of $\mathrm{SH}$ waves in an irregular non homogeneous monoclinic crustal layer over a semi-infinite monoclinic medium, Appl. Math. Sci. 4, 44, 2157-2170.

Chattopadhyay, A., S. Gupta, and A.K. Singh (2010b), The dispersion of shear wave in multi-layered magnetoelastic self-reinforced media, Int. J. Solids Struct. 47, 9, 1317-1324, DOI: 10.1016/j.ijsolstr.2010.01.019.

Chattopadhyay, A., S. Gupta, V.K. Sharma, and P. Kumari (2010c), Propagation of shear waves in viscoelastic medium at irregular boundaries, Acta Geophys. 58, 2, 195-214, DOI: 10.2478/s11600-009-0060-3.

Chattopadhyay, A., S. Gupta, S.A. Sahu, and A.K. Singh (2013), Dispersion of horizontally polarized shear waves in an irregular non-homogeneous selfreinforced crustal layer over a semi-infinite self-reinforced medium, $J$. Vib. Control 19, 1, 109-119, DOI: 10.1177/1077546311430699.

Du, J., X. Jin, and J. Wang (2007), Love wave propagation in layered magnetoelectro-elastic structures with initial stress, Acta Mechanica 192, 1-4, 169189, DOI: 10.1007/s00707-006-0435-3.

Ewing, W.M., W.S. Jardetzky, and F. Press (1957), Elastic Wave in Layered Media, McGraw-Hill, New York.

Gubbins, D. (1990), Seismology and Plate Tectonics, Cambridge University Press, Cambridge, 170 pp.

Kaur, J., S.K. Tomar, and V.P. Kaushik (2005), Reflection and refraction of SHwaves at a corrugated interface between two laterally and vertically heterogeneous viscoelastic solid half-spaces, Int. J. Solids Struct. 42, 13, 36213643, DOI: 10.1016/j.ijsolstr.2004.11.014.

Ke, L.L., Y.S. Wang, and Z.M. Zhang (2005), Propagation of Love waves in an inhomogeneous fluid saturated porous layered half-space with properties varying exponentially, J. Eng. Mech. 131, 12, 1322-1328, DOI: 10.1061/ (ASCE)0733-9399(2005)131:12(1322).

Ke, L.L., Y.S. Wang, and Z.M. Zhang (2006), Propagation of Love waves in an inhomogeneous fluid saturated porous layered half-space with linearly vary- 
ing properties, Soil. Dyn. Earthq. Eng. 26, 6-7, 574-581, DOI: 10.1016/ j.soildyn.2006.01.010.

Kundu, S., S. Manna, and S. Gupta (2014), Love wave dispersion in pre-stressed homogeneous medium over a porous half-space with irregular boundary surfaces, Int. J. Solids Struct. 51, 21-22, 3689-3697, DOI: 10.1016/j.ijsolstr. 2014.07.002.

Markham, M.F. (1970), Measurements of elastic constants of fibre composites by ultrasonics, Composites 1, 2, 145-149, DOI: 10.1016/0010-4361(69)90059-7.

Samal, S.K., and R. Chattaraj (2011), Surface wave propagation in fibre-reinforced anisotropic elastic layer between liquid saturated porous half-space and uniform liquid layer, Acta Geophys. 59, 3, 470-482, DOI: 10.2478/s11600011-0002-8.

Sengupta, P.R., and S. Nath (2001), Surface waves in fibre-reinforced anisotropic elastic media, Sadhana 26, 4, 363-370, DOI: 10.1007/BF02703405.

Singh, B., and S.J. Singh (2004), Reflection of plane waves at the free surface of a fibre-reinforced elastic half-space, Sadhana 29, 3, 249-257, DOI: 10.1007/BF02703774.

Singh, S.S. (2011), Love wave at a layer medium bounded by irregular boundary surfaces, J. Vib. Control 17, 5, 789-795, DOI: 10.1177/1077546309351301.

Singh, S.S., and S.K. Tomar (2008), qP-wave at a corrugated interface between two dissimilar pre-stressed elastic half-spaces, J. Sound Vib. 317, 3-5, 687-708, DOI: 10.1016/j.jsv.2008.03.036.

Son, M.S., and Y.J. Kang (2012), Propagation of shear waves in a poroelastic layer constrained between two elastic layers, Appl. Math. Model. 36, 8, 36853695, DOI: 10.1016/j.apm.2011.11.008.

Tomar, S.K., and J. Kaur (2007), SH-waves at a corrugated interface between a dry sandy half-space and an anisotropic elastic half-space, Acta Mech. 190, 1-4, 1-28, DOI: 10.1007/s00707-006-0423-7.

Wolf, B. (1970), Propagation of Love waves in layers with irregular boundaries, Pure Appl. Geophys. 78, 1, 48-57, DOI: 10.1007/BF00874772.

Received 18 February 2015

Received in revised form 12 November 2015

Accepted 19 November 2015 